\title{
A Novel Machine Learning-Based Handover Scheme for Hybrid LiFi and WiFi Networks
}

\author{
Xiping Wu and Dominic C. O'Brien \\ Department of Engineering Science \\ University of Oxford, Oxford, OX1 3PJ, UK \\ \{xiping.wu, dominic.obrien\}@eng.ox.ac.uk
}

\begin{abstract}
Combining the high area spectrum efficiency of light fidelity ( $\mathrm{LiFi}$ ) and the ubiquitous coverage of wireless fidelity (WiFi), hybrid LiFi and WiFi networks have drawn increasing research attention. Meanwhile, the handover issue in hybrid networks becomes a hotspot since the coverage areas of $\mathrm{LiFi}$ and WiFi overlap each other. In addition, LiFi may cause frequent handovers for fast-moving users, while $\mathrm{WiFi}$ is susceptible to traffic overload. Consequently, the selection between $\mathrm{LiFi}$ and WiFi becomes a tricky problem. In this paper we propose a novel handover scheme, which adopts a dynamic coefficient via machine learning to adjust the selection preference between $\mathrm{LiFi}$ and WiFi. The new method balances channel quality, resource availability and user mobility to make handover decisions. Results show that compared to the received signal strength (RSS)-based and trajectory-based handover methods, the proposed scheme can improve the user's throughput by up to $260 \%$ and $50 \%$, respectively.
\end{abstract}

Index Terms-Hybrid network, light fidelity (LiFi), optical wireless communications (OWC), handover, machine learning, artificial neural network

\section{INTRODUCTION}

$\mathrm{I}_{\mathrm{i}}^{\mathrm{N}}$ NDOOR wireless communications are facing exponentially increasing demands on data traffic, which will increase 3 -fold from 2017 to 2020 [1]. As a promising solution to future indoor wireless communications, hybrid $\mathrm{LiFi}$ and $\mathrm{WiFi}$ networks have recently attracted increasing research attention [2]. Such a network combines the ubiquitous coverage of $\mathrm{WiFi}$ and the high area spectrum efficiency of $\mathrm{LiFi}$, which uses light wave as signal bearers. Compared with WiFi, LiFi offers a number of advantages including: i) huge and unlicensed optical spectrum, ii) availability in radio frequency (RF)restricted areas, iii) provision of illumination and iv) secure communications.

While the hybrid network can substantially improve indoor network performance, handover becomes a challenging issue. This is mainly due to three factors. First, the coverage range of a single LiFi access point (AP) is relatively small, usually with a 2-3 m diameter. As a result, frequent handovers might occur to mobile users even with a walking speed. Second, the coverage areas of $\mathrm{LiFi}$ and $\mathrm{WiFi}$ overlap each other. This makes the handover methods that only rely on channel quality less effective. Third, WiFi is susceptible to traffic overload since a WiFi AP has a larger coverage area than a LiFi AP. Consequently, resource availability needs to be taken into account to balance the traffic loads. In summary, when executing handovers in a hybrid LiFi and WiFi network, it is necessary to jointly consider channel quality, resource availability and user mobility. Therefore, it is tricky to make a decision between horizontal handover (HHO) and vertical handover (VHO), which refer to the handovers occurring between two APs with the same network technology and with different network technologies, respectively.

A significant number of relevant studies have been carried out. In [3], a trajectory-based handover scheme was proposed for ultra-dense networks. This type of method allows handovers to take place between two non-adjacent APs but relies on the user's trajectory information. Associating AP selection with resource allocation, optimisation-based load balancing methods were developed [4], with enormously high computational complexity. To reduce the processing power, researchers attempted to apply game theory in [5] and fuzzy logic in [6]. However, [5] requires quantities of iterations to reach a steady state, whereas [6] can only provide suboptimal solutions. Moreover, [4]-[6] fail to accommodate the impact of user mobility. In [7], the concept of mobility-aware load balancing was introduced. This method takes the handover cost into account when maximising network throughput, while still needing considerable computational complexity.

In this paper we investigate the use of machine learning in the handover process for hybrid networks. So far, a handful of research work has been conducted in this field. In [8], an artificial neural network (ANN)-based handover method was developed for heterogeneous networks. Also using an ANN, a quality of experience (QoE)-based vertical handover scheme was proposed in [9]. These studies have two limitations: i) they only manage VHOs; and ii) resource availability is not considered. Motivated by this, we propose a novel ANN-based handover scheme which takes into account channel quality, resource availability and user mobility. Unlike [8] and [9] using ANNs to directly make handover decisions, the new approach employs an ANN to train a dynamic coefficient which adjusts the selection preference between LiFi and WiFi. To the best of our knowledge, the proposed handover scheme is the first of its kind in the existing literature.

The remainder of this paper is organised as follows. The system model is introduced in Section II, including network deployment, channel models and light-path blockages. The novel handover scheme is proposed in Section III. Simulation results are presented in Section IV. Finally, conclusions are drawn in Section V. 


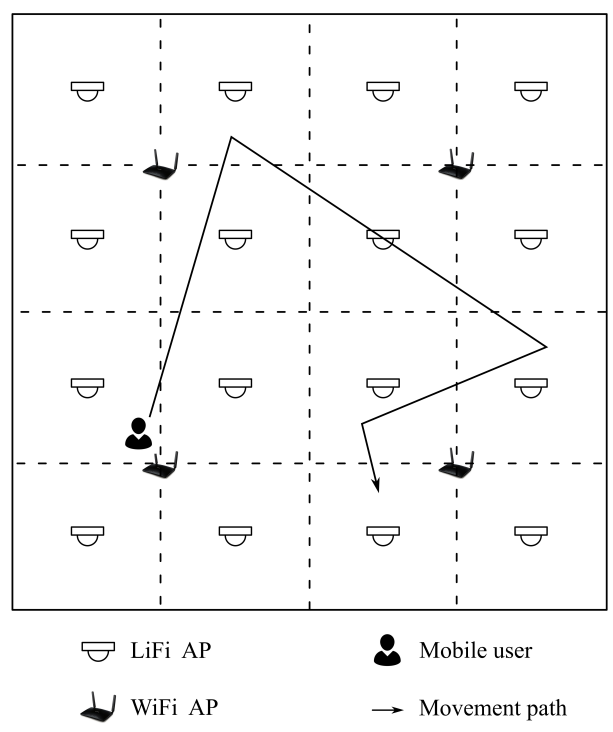

Fig. 1. Schematic diagram of an indoor HLWNet.

\section{System ModeL}

Fig. 1 shows the schematic diagram of an indoor HLWNet. A number of LiFi APs are located in the coverage area of each WiFi AP. The LiFi APs are arranged in a lattice topology, with each one operating on a ceiling LED lamp. The WiFi system uses carrier sense multiple access/collision avoidance to keep inter-cell interference (ICI) at an undetectable level. As for $\mathrm{LiFi}$, frequency reuse with a factor of 4 is applied to mitigate ICI. The users are assumed to move randomly by following the random waypoint (RWP) model [10]. Time-division multiple accessing (TDMA) is employed to enable the APs to serve multiple users.

\section{A. LiFi Channel Model}

A LiFi channel consists of two components: line-of-sight (LoS) and non line-of-sight (NLoS) paths. Let $d_{i, u}$ denote the Euclidean distance of the LoS path between AP $i$ and user $u$. Let $\phi_{i, u}$ and $\psi_{i, u}$ denote the angles of irradiance and incidence, respectively. The LoS channel gain can be expressed as [11, eq. (10)]:

$$
H_{i, u}^{\mathrm{LoS}}=\frac{(m+1) A_{\mathrm{pd}}}{2 \pi d_{i, u}^{2}} \cos ^{m}\left(\phi_{i, u}\right) g_{f} g_{c}\left(\psi_{i, u}\right) \cos \left(\psi_{i, u}\right),
$$

where $m=-\ln 2 / \ln \left(\cos \Phi_{1 / 2}\right)$ is the Lambertian emission order, and $\Phi_{1 / 2}$ is the angle of half intensity; $A_{\mathrm{pd}}$ is the physical area of the photodiode (PD); $g_{f}$ is the optical filter gain; $g_{c}\left(\psi_{i, u}\right)$ denotes the optical concentrator gain, which is given by:

$$
g_{c}\left(\psi_{i, u}\right)=\left\{\begin{array}{ll}
\frac{n^{2}}{\sin ^{2}\left(\Psi_{\max }\right)}, & 0 \leq \psi_{i, u} \leq \Psi_{\max } \\
0, & \psi_{i, u}>\Psi_{\max }
\end{array},\right.
$$

where $n$ represents the refractive index, and $\Psi_{\max }$ is the semiangle of the field of view (FoV) of the PD.
Only first-order reflections are considered for the NLoS paths, since higher-order reflections typically contribute little [11]. The NLoS channel gain is denoted by $H_{i, u}^{\mathrm{NLoS}}$, which is expressed in (3). The total gain of a LiFi channel is $H_{i, u}=H_{i, u}^{\mathrm{LoS}}+H_{i, u}^{\mathrm{NLoS}}$. At the receiver, a PD captures photons and converts them into a photocurrent:

$$
I=R_{\mathrm{pd}} H_{i, u} P_{\mathrm{mod}}
$$

where $R_{\mathrm{pd}}$ denotes the detector responsivity and $P_{\text {mod }}$ is the average modulated optical power. The signal-to-interferenceplus-noise ratio (SINR) of the LiFi link between AP $i$ and user $u$ is written as follows:

$$
\gamma_{\mathrm{LiFi}}^{i, u}=\frac{\left(R_{\mathrm{pd}} H_{i, u} P_{\mathrm{mod}}\right)^{2}}{N_{\mathrm{LiFi}} B_{\mathrm{LiFi}}+\sum_{j \in \mathcal{I}, j \neq i}\left(R_{\mathrm{pd}} H_{j, u} P_{\mathrm{mod}}\right)^{2}},
$$

where $B_{\mathrm{LiFi}}$ is the bandwidth of the LiFi AP; $N_{\mathrm{LiFi}}$ denotes the power spectral density (PSD) of noise, which is assumed to be signal independent; and $\mathcal{I}$ is the set of APs that employ the same optical spectrum as AP $i$.

\section{B. WiFi Channel Model}

The gain of a WiFi channel is given by [12]:

$$
G_{\mathrm{WiFi}}^{i, u}=\left|H_{\mathrm{WiFi}}^{i, u}\right|^{2} 10 \frac{-L\left(d_{i, u}\right)+X_{\sigma}}{10},
$$

where $H_{\mathrm{WiFi}}^{i, u}$ defines the channel transfer function, which follows a standard Rayleigh distribution; the shadow fading $X_{\sigma}$ is a zero-mean Gaussian random variable with a standard deviation of $10 \mathrm{~dB}$; and $L(\cdot)$ denotes the free-space path loss:

$$
L(d)=\left\{\begin{array}{ll}
20 \log _{10}\left(f_{c} d\right)-147.5, & d<d_{\mathrm{ref}} \\
20 \log _{10}\left(f_{c} \frac{d^{2.75}}{d_{\mathrm{ref}}^{1.75}}\right)-147.5, & d \geq d_{\mathrm{ref}}
\end{array},\right.
$$

where $f_{c}$ is the central carrier frequency and $d_{\text {ref }}=10 \mathrm{~m}$ is the reference distance. The SINR of a WiFi link can then be written as follows:

$$
\gamma_{\mathrm{WiFi}}^{i, u}=\frac{G_{\mathrm{WiFi}}^{i, u} P_{\mathrm{WiFi}}}{N_{\mathrm{WiFi}} B_{\mathrm{WiFi}}}
$$

where $N_{\mathrm{WiFi}}$ is the PSD of noise at the receiver; $B_{\mathrm{WiFi}}$ and $P_{\mathrm{WiFi}}$ denote the system bandwidth and transmit power of the WiFi AP, respectively.

\section{Light-path Blockage}

Two parameters are considered to model the light-path blockages: occurrence rate and occupation rate [7]. The occurrence rate is defined as the average number of blockages that occur in a time unit. In queueing theory, the Poisson point process (PPP) is widely used to model random events such as the arrival of packets at a switch. Here the blockage events are also assumed to follow the PPP. The occupation rate is defined as the amount of time that is occupied by blockages during a time unit. This parameter is assumed to be uniformly distributed between 0 and 1 . 


$$
H_{\mathrm{NLoS}}^{i, u}=\int_{A_{w}} \frac{(m+1) A_{\mathrm{pd}}}{2\left(\pi d_{i, w} d_{w, u}\right)^{2}} \rho_{w} \cos ^{m}\left(\phi_{i, w}\right) g_{f} g_{c}\left(\psi_{w, u}\right) \cos \left(\psi_{w, u}\right) \cos \left(\vartheta_{i, w}\right) \cos \left(\vartheta_{w, u}\right) d A_{w}
$$

\section{Achievable Data Rate}

The WiFi capacity can be measured by Shannon capacity. Unfortunately, this does not apply to LiFi since $\gamma_{\mathrm{LiFi}}^{i, u}$ is an electrical SINR for non-negative signals. A lower bound of the LiFi channel was derived in [13], which can be used for $\gamma_{\mathrm{LiFi}}^{i, u}$. Regarding the link between AP $i$ and user $u$, the achievable data rate can be computed as follows:

$$
r_{i, u}= \begin{cases}\rho_{i, u} \frac{B_{i}}{2} \log _{2}\left(1+\frac{e}{2 \pi} \gamma_{\mathrm{LiFi}}^{i, u}\right), & \text { for a LiFi AP } \\ \rho_{i, u} B_{i} \log _{2}\left(1+\gamma_{\mathrm{WiFi}}^{i, u}\right), & \text { for a WiFi AP }\end{cases}
$$

where $\rho_{i, u}$ denotes the proportion of time resource that AP $i$ allocates to user $u$. The parameter $\rho_{i, u}$ can be determined by a proportional fairness scheduler in [6, eq. (13)].

\section{Proposed Handover Scheme}

In this section, a novel handover scheme is proposed to jointly handle channel quality, resource availability and user mobility. Specifically, a dynamic coefficient is introduced to adjust the selection preference between LiFi and WiFi. This coefficient is trained by an ANN to fit different scenarios.

\section{A. Handover Scheme}

Our previous work in [14] has shown that the rate of change in RSS can indicate whether the user is moving towards the centre of an AP. Combining RSS and its rate of change can effectively reduce unnecessary handovers by skipping certain APs. Taking resource availability into account, the objective function of the proposed handover scheme is formulated as follows:

$$
\Gamma_{i}=\left\{\begin{array}{ll}
\zeta_{i}\left(\gamma_{i}+\Delta \gamma_{i}\right), & \text { if } i \text { is a LiFi AP } \\
\lambda \zeta_{i}\left(\gamma_{i}+\Delta \gamma_{i}\right), & \text { if } i \text { is a WiFi AP }
\end{array},\right.
$$

where $\zeta_{i}$ denotes the user's satisfaction degree, which in this paper is defined as the ratio of the achievable data rate to the required data rate; $\gamma_{i}$ indicates the RSS, whereas $\Delta \gamma_{i}$ represents its change of rate. The three above parameters reflect the impact of resource availability, channel quality and user mobility on handover decisions, respectively. The coefficient $\lambda$ is used to indicate the selection preference between LiFi and WiFi in the hybrid network. (See details about this coefficient in Section III-B.)

The handover algorithm in long term evolution (LTE) [15] employs the concept of hysteresis to mitigate the ping-pong effect. Specifically, the making-decision process is triggered when the RSS of the host AP is below that of the target AP. But the handover decision is not immediately made until the target AP keeps providing a higher RSS for a certain amount of time, with a typical value of hundreds of milliseconds [15]. Here we use a similar mechanism but with dual triggers: RSS and the user's satisfaction degree. When either of the two

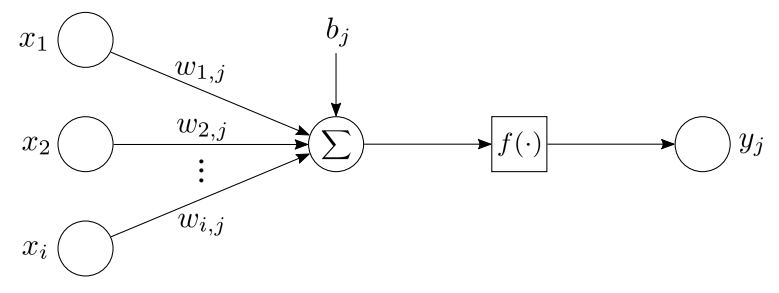

Fig. 2. The basic structure of a neuron.

parameters related to the host $\mathrm{AP}$ is below any other AP, the proposed handover scheme starts to count time. The time counter continues as long as the trigger condition is met, and otherwise is reset. When the counted time reaches the preset threshold, a handover decision is made to transfer the user from the host AP to the target AP that provides the highest value of $\Gamma_{i}$.

\section{B. Machine Learning Algorithm}

Now we discuss how to determine the coefficient $\lambda$. In general, fast-moving users prefer WiFi to avoid frequent handovers between LiFi APs. In this case, $\lambda$ should be a number larger than 1 to increase the value of $\Gamma_{i}$ for $\mathrm{WiFi}$ APs. When the user is static or moves very slowly, $\lambda$ should be 1 to let the user choose the AP that provides the highest value of $\zeta_{i} \gamma_{i}$. This means that for a static user, the different coverage ranges of LiFi and WiFi do not affect the handover decision. The optimal value of $\lambda$ is dependent on a number of factors including the user's speed (denoted by $V$ ), the separation between two nearest LiFi APs (denoted by $S$ ), the height of LiFi APs (denoted by $H$ ), and the number of LiFi APs per WiFi AP (denoted by $N$ ). The choice of $\lambda$ can be deemed as a function of these factors. However, due to the complicated process of making handover decisions, it is not feasible to derive a closed-form expression for this function. Therefore, we propose to determine $\lambda$ with ANN.

Fig. 2 depicts the basic structure of a neuron. There are a number of inputs, with $x_{i}$ denoting the $i$-th input. The output of the $j$-th neuron is denoted by $y_{j}$. For each pair of input and output, there is a weight which is denoted by $w_{i, j}$. A bias $b_{j}$ is added to the weighted sum of inputs. Apart from that, an activation function $f(\cdot)$ is used to introduce non-linearity into the output of a neuron. Here the activation function is chosen to be tanh, which is widely used in ANNs. The output of a neuron can be expressed as follows:

$$
y_{j}=\tanh \left(b_{j}+\sum_{i} x_{i} w_{i, j}\right) .
$$

Based on the neuron structure, the ANN model of $\lambda$ can be constructed as shown in Fig. 3. Let $h_{j}$ denote the $j$-th node in the hidden layer. The notation of weights is modified to 


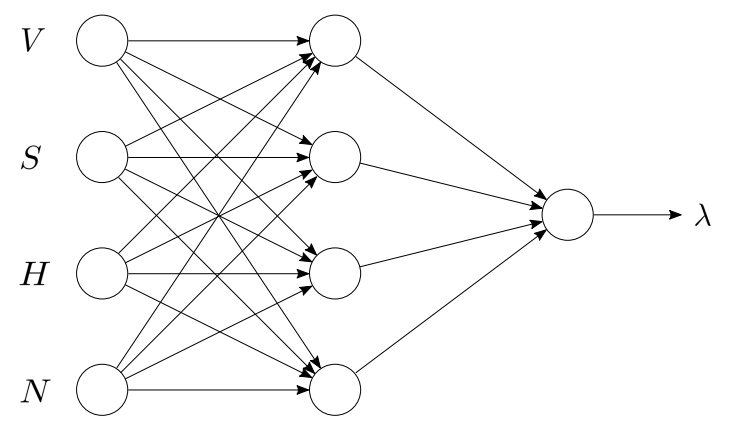

Input layer Hidden layer Output layer

Fig. 3. The ANN model of $\lambda$.

$w_{k, i, j}$, where $k$ indicates different layers. Similarly, the biases pertaining to the hidden nodes are denoted by $b_{1, j}$, while the bias added to the output is denoted by $b_{2}$. The ANN output can be computed by:

$$
\lambda=\tanh \left[b_{2}+\sum_{j} \tanh \left(b_{1, j}+\sum_{i} x_{i} w_{1, i, j}\right) w_{2, j}\right] .
$$

\section{Training and Test}

Given inputs, the optimal solution (denoted by $\hat{\lambda}$ ) can be obtained numerically by measuring the average throughput with different values of $\lambda$. It is worth noting that the output range is restricted between -1 and 1 due to the function tanh. As a result, these optimal solutions need to be normalised before constituting the sample set for training and validation. A 75:25 ratio between the training data and the test data is adopted. In other words, we randomly choose $3 / 4$ of the samples to train the ANN system and use the remaining samples to measure the fitness. Let $M$ denote the number of training samples. The loss function of the ANN system can be calculated by:

$$
L=\frac{1}{M} \sum_{m=1}^{M}\left(\lambda_{m}-\hat{\lambda}_{m}\right)^{2} .
$$

The gradient descent method is used to update the weights and biases on each iteration:

$$
w_{k, i, j}=w_{k, i, j}-\eta \frac{\partial L}{\partial w_{k, i, j}},
$$

where $\eta$ is the learning rate, which controls the step of iterations. The backpropagation method is used to reduce computational complexity. Specifically, the parameters are updated in the sequence of $b_{2}, w_{2, j}, b_{1, j}$ and $w_{1, i, j}$.

\section{Simulation Results}

Monte Carlo simulations are implemented to evaluate the performance of the proposed method. Two baselines are considered: the standard LTE handover scheme (referred to as STD) and the trajectory-based handover method [3]. All methods use the same time-to-trigger of $160 \mathrm{~ms}$ [15], to
TABLE I

SimUlation PARAMETERS

\begin{tabular}{l|l}
\hline Parameter & Value \\
\hline The physical area of the PD, $A_{\mathrm{pd}}$ & $1 \mathrm{~cm}^{2}$ \\
Optical filter gain, $g_{f}$ & 1 \\
Refractive index, $n$ & 1.5 \\
Half-intensity radiation angle, $\Phi_{1 / 2}$ & $60^{\circ}$ \\
FoV semi-angle of the PD, $\Psi_{\max }$ & $90^{\circ}$ \\
Detector responsivity, $R_{\mathrm{pd}}$ & $0.53 \mathrm{~A} / \mathrm{W}$ \\
Wall reflectivity & 0.8 \\
Modulated optical power per LiFi AP, $P_{\mathrm{mod}}$ & $1 \mathrm{Watt}$ \\
Transmitted power per WiFi AP, $P_{\mathrm{WiFi}}$ & $20 \mathrm{dBm}$ \\
Bandwidth per LiFi AP, $B_{\mathrm{LiFi}}$ & $20 \mathrm{MHz}$ \\
Bandwidth per WiFi AP, $B_{\mathrm{WiFi}}$ & $20 \mathrm{MHz}$ \\
PSD of noise in LiFi, $N_{\mathrm{LiFi}}$ & $10^{-21} \mathrm{~A}^{2} / \mathrm{Hz}[7]$ \\
PSD of noise in WiFi, $N_{\mathrm{WiFi}}$ & $-174 \mathrm{dBm} / \mathrm{Hz}[7]$ \\
\hline
\end{tabular}
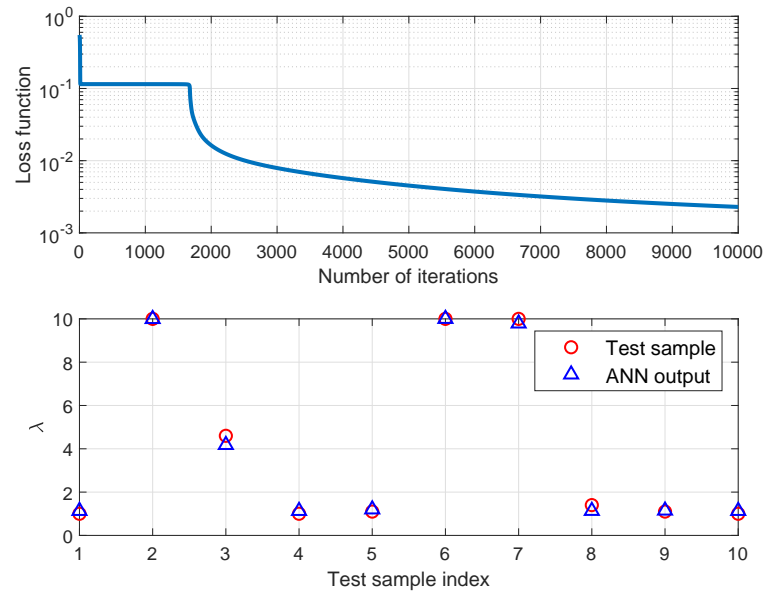

Fig. 4. Training and test results of the ANN model.

provide a fair comparison. The average overhead of HHO is about $200 \mathrm{~ms}$ in wireless local area networks (WLANs), while the average overhead of VHO is set to be $500 \mathrm{~ms}$ [7]. Other parameters are summarised in Table I.

The ANN model is first validated. The initial weights and biases are small random numbers, while the learning rate is set to be 0.01. Fig. 4 presents the training and test results of the ANN model. We use 75 training samples with different combinations of $V, S, H$ and $N$. As the number of iterations increases, the loss function decreases gradually and reaches $2 \times 10^{-3}$ with 10000 iterations. After training, the ANN model is validated with 25 test samples. As shown, the ANN outputs well match the expected results, with a mean squared error below $4 \times 10^{-3}$.

Now we evaluate the performance of the proposed method in different scenarios, with the dynamic coefficient $\lambda$ yielded by the trained ANN model. Fig. 5 shows the average achievable throughput as a function of the user's speed, while light-path blockages are not involved here. As can be seen, all handover methods provide almost the same throughput when the user's speed is low. However, as the user's speed 


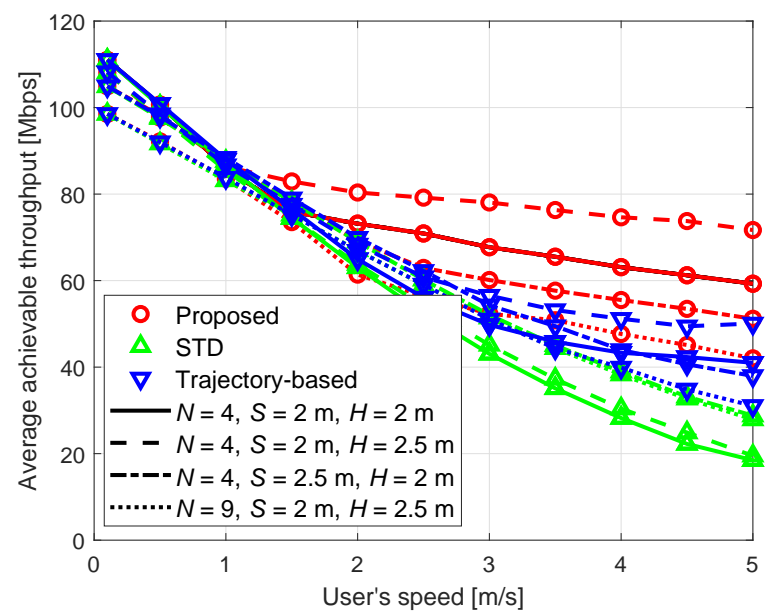

Fig. 5. Average achievable throughput versus the user's speed.

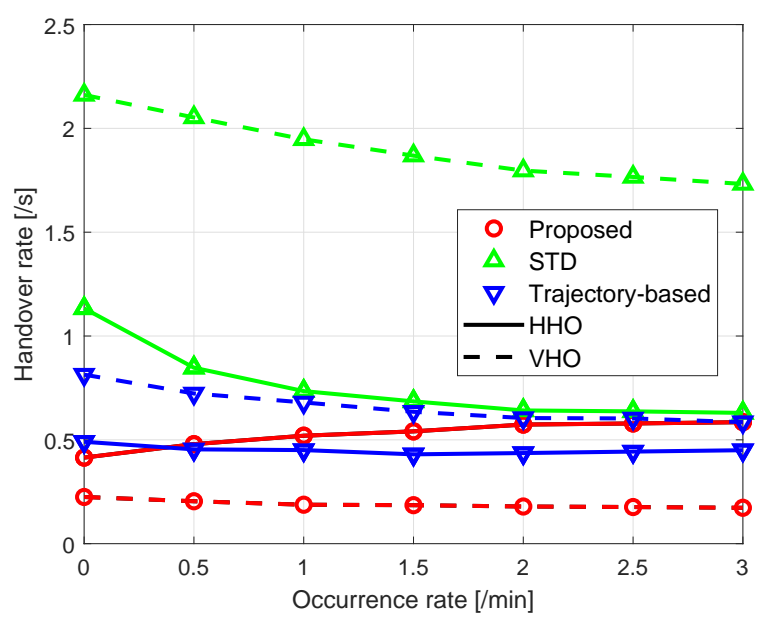

Fig. 6. Handover rates of $\mathrm{HHO}$ and VHO.

increases, the proposed method achieves a significantly higher throughput than the baseline methods. This is attributed to the dynamic network selection preference. Also, it is observed that the throughput improvement becomes larger for a $N$, a $S$, and a $H$. When $V=5 \mathrm{~m} / \mathrm{s}, N=4, S=2 \mathrm{~m}$ and $H=2.5 \mathrm{~m}$, the proposed scheme supports an average throughput of $72 \mathrm{Mbps}$, which is about $260 \%$ higher than STD and 50\% higher than the trajectory-based method.

Finally, the handover rates of $\mathrm{HHO}$ and VHO are measured in different situations of light-path blockages. The scenario of $V=5 \mathrm{~m} / \mathrm{s}, N=4, S=2 \mathrm{~m}, H=2.5 \mathrm{~m}$ is taken as an example. As shown in Fig. 6, the proposed method can effectively suppress frequent VHOs in comparison to the baseline methods. When the occurrence rate is 3 times per minute, for example, the proposed method can reduce the overall handover rate by $68 \%$ and $27 \%$ against STD and the trajectory-based approach, respectively.

\section{Conclusions}

In this paper, a novel machine learning-based handover scheme was proposed for hybrid $\mathrm{LiFi}$ and $\mathrm{WiFi}$ networks. This approach takes into account channel quality, resource availability and user mobility, and adopts a dynamic coefficient to adjust the selection preference between $\mathrm{LiFi}$ and $\mathrm{WiFi}$. This coefficient is trained through ANN for different scenarios including the user's speed, the separation between LiFi APs, the height of LiFi APs, and the number ratio between $\mathrm{LiFi}$ APs and WiFi APs. Simulations were carried out to validate the ANN model and evaluate the performance of the proposed handover scheme. Results show that the new method can effectively avoid frequent handovers, with a handover rate $68 \%$ less than STD and 27\% less than the trajectory-based method. In terms of achievable throughput, the proposed scheme outperforms the two baselines by up to $260 \%$ and $50 \%$, respectively. Future work will aim to reduce the complexity of the ANN model and study its rate of convergence.

\section{ACKNOWLEDGEMENT}

This work was supported by the European Union's Horizon 2020 research and innovation programme under grant agreement No 825651 (ELIoT).

\section{REFERENCES}

[1] "Cisco visual networking index: Forecast and trends, 2017-2022," Cisco, San Jose, CA, USA, Tech. Rep., Nov. 2018.

[2] X. Wu et al., "Hybrid LiFi and WiFi networks: A survey," arXiv preprint arXiv:2001.04840, 2020.

[3] R. Arshad et al., "Handover management in 5G and beyond: A topology aware skipping approach," IEEE Access, vol. 4, pp. 9073-9081, 2016.

[4] X. Li, R. Zhang, and L. Hanzo, "Cooperative load balancing in hybrid visible light communications and WiFi," IEEE Trans. Commun., vol. 63, no. 4, pp. 1319-1329, Apr. 2015.

[5] Y. Wang, $\mathrm{X}$. Wu, and $\mathrm{H}$. Haas, "Load balancing game with shadowing effect for indoor hybrid LiFi/RF networks," IEEE Trans. Wireless Commun., vol. 16, no. 4, pp. 2366-2378, Apr. 2017.

[6] X. Wu, M. Safari, and H. Haas, "Access point selection for hybrid LiFi and Wi-Fi networks," IEEE Trans. Commun., vol. 65, no. 12, pp. 5375-5385, Dec. 2017.

[7] X. Wu and H. Haas, "Load balancing for hybrid LiFi and WiFi networks: To tackle user mobility and light-path blockage," IEEE Trans. Commun., vol. 68 , no. 3, pp. 1675-1683, Mar. 2020.

[8] N. M. Alotaibi and S. S. Alwakeel, "A neural network based handover management strategy for heterogeneous networks," in IEEE 14th Int. Conf. on Machine Learning and Applications (ICMLA), Miami, FL, 2015, pp. 1210-1214.

[9] A. B. Zineb et al., "QoE-based vertical handover decision management for cognitive networks using ANN," in 6th Int. Conf. on Communications and Networking (ComNet), Hammamet, 2017, pp. 1-7.

[10] D. Johnson and D. Maltz, "Dynamic source routing in ad hoc wireless networks," Mobile Computing, pp. 153-181, 1996.

[11] J. Kahn and J. Barry, "Wireless infrared communications," Proc. IEEE, vol. 85, no. 2, pp. 265-298, Feb. 1997.

[12] E. Perahia and R. Stacey, Next Generation Wireless LAN: 802.11n and 802.11ac. Cambridge, U.K.: Cambridge University Press, 2013.

[13] J. Wang, Q. Hu, J. Wang, M. Chen, and J. Wang, "Tight bounds on channel capacity for dimmable visible light communications," J. Lightw. Technol., vol. 31, no. 23, pp. 3771-3779, Dec. 2013.

[14] X. Wu and D. C. O'Brien, "A novel handover scheme for hybrid LiFi and WiFi networks," in 2020 IEEE Int. Conf. on Communications (ICC), Dublin, 2020, pp. 1-5.

[15] "LTE; Evolved universal terrestrial radio access (E-UTRA); Radio resource control (RCC); Protocol specification (Release 13)," 3GPP TS 36.331 v13.0.0., Valbonne, France, Tech. Rep., Jan. 2016. 